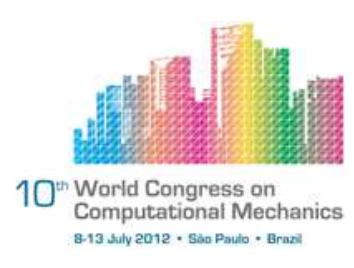

\title{
COMPUTATION OF MOMENTS IN ORTHOTROPIC THICK PLATES BY THE BOUNDARY ELEMENT METHOD
}

\author{
A. Reis ${ }^{1}$, E. L. Albuquerque ${ }^{1}$, A. P. Santana ${ }^{2}$, J. F. Useche ${ }^{3}$ \\ ${ }^{1}$ Faculty of Technology, Department of Mechanical Engineering, University of Brasília - UnB \\ (eder@unb.br) \\ ${ }^{2}$ Department of Mechanical and Materials, Federal Institute of Maranhão - IFMA \\ ${ }^{3}$ Faculty of Mechanical Engineering, University Technological of Bolivar
}

\begin{abstract}
This paper presents a boundary element formulation for the computation of moments at internal points of laminated composite thick plates. Fundamental solutions for orthotropic thick plates are obtained using Hörmander operator and Radon transform. So, they do not have a closed form and numerical integration is necessary to compute fundamental solutions in each field point. Integral equations for moments are developed and all derivatives of the fundamental solution are computed. A special integration technique is used in order to improve performance of the method. The obtained results are in good agreement with literature.
\end{abstract}

Keywords: Orthotropic thick plates, boundary element method, Radon transform, numerical integration.

\section{INTRODUCTION}

In recent years, the boundary element method (BEM) has become an attractive tool for resolution of complex problems which have the formulation described by partial differential equations. Analysis of plate bending problems using the BEM has attracted the attention of many researchers during the past years, proving to be a particularly adequate field of applications for that technique. The first work about thick Reissner and Mindlin plates using boundary method element has been proposed by [1]. Many works have reported the application of boundary method elements to bending analysis in thick plates using the theory Reissner [2], [3], [4], [7], [9] and [10]. [8] presented a boundary method element formulation to thick Mindlin plates for orthotropic shear deformable plates. This work proposes a numerical procedure to compute moments at internal points of orthotropic shear deformable plates using a boundary element formulation that follows the Mindlin hypotheses. It uses the fundamental solution proposed in [8] that takes into account the effects of shear deformation and was derived by means of Hörmander operator and the Radon transform. Some numerical examples concerning orthotropic plate bending problems are analyzed with the BEM. 


\section{MINDLIN PLATE THEORY}

The Mindlin's theory assumes displacement distribution through the thickness. Using the assumptions of the classical theory, he removed the hypothesis of the transverse shear deformation equal zero in the mid plane, but considering that distortion variation is null. Thus:

$$
\begin{aligned}
& \frac{\partial \gamma_{13}}{\partial x_{3}}=0 . \\
& \frac{\partial \gamma_{23}}{\partial x_{3}}=0 .
\end{aligned}
$$

So, equations of equilibrium for the plate are given by:

$$
\begin{gathered}
M_{\alpha \beta, \beta}-Q_{\alpha}=0 . \\
Q_{\alpha, \alpha}+q=0 .
\end{gathered}
$$

where $q$ is the distributed transverse load per unit area in the $x_{3}$ direction. The bending moments $M_{\alpha \beta}$ and the shear forces $Q_{\alpha}$ for orthotropic plates are expressed in terms of the rotations and the lateral displacement as:

$$
\begin{gathered}
M_{\alpha \beta}=D_{\alpha \beta}\left(w_{\alpha, \beta}+w_{\beta, \alpha}\right)+C_{\alpha \beta} w_{\gamma, \gamma} . \\
Q_{\alpha}=C_{\alpha}\left(w_{\alpha}+w_{3, \alpha}\right) .
\end{gathered}
$$

where no summation is assumed in Eq. (5) and Eq. (6) with respect to the indices $\alpha, \beta$. Material parameters are gives as [11]:

$$
\begin{array}{lll}
D_{11}=\frac{D_{1}}{2}\left(1-\nu_{21}\right), & D_{22}=\frac{D_{2}}{2}\left(1-\nu_{12}\right), & D_{12}=D_{21}=D_{k}=\frac{G_{12} h^{3}}{12}, \\
C_{11}=D_{1} \nu_{21}, & C_{22}=D_{2} \nu_{12}, & C_{12} C_{21}=0, \\
D_{1}=\frac{E_{1} h^{3}}{12\left(1-\nu_{12} \nu_{21}\right)}, & D_{2}=\frac{E_{2} h^{3}}{12\left(1-\nu_{12} \nu_{21}\right)}, & D_{1} \nu_{21}=D_{2} \nu_{12}, \\
C_{1}=G_{13} k h \quad, & C_{2}=G_{23} k h . &
\end{array}
$$

in which $k=5 / 6$ in the Reissner plate theory, $E_{1}$ and $E_{2}$ represent Young's moduli, $G_{12}, G_{13}$ and $G_{23}$ are shear moduli, $\nu_{12}$ and $\nu_{21}$ are Poisson's ratios, respectively, and $h$ is the thickness of the plate.

\section{DIFFERENTIAL EQUATIONS OF EQUILIBRIUM}

The differential equation of equilibrium is give by [8]:

$$
L_{i j} U_{j}+b_{i}=0
$$


where $b_{i}$ represent the body force and $L_{i j}$ are Navier differential operators, which can be written as:

$$
\begin{array}{cc}
L_{11}=D_{1} \frac{\partial^{2}}{\partial x_{1}^{2}}+D_{k} \frac{\partial^{2}}{\partial x_{2}^{2}}-C_{1}, \quad L_{22}=D_{k} \frac{\partial^{2}}{\partial x_{1}^{2}}+D_{2} \frac{\partial^{2}}{\partial x_{2}^{2}}-C_{2}, \\
L_{12}=L_{21}=\left(D_{1} \mu_{y x}+D_{k}\right) \frac{\partial^{2}}{\partial x_{1} \partial x_{2}}, \quad L_{13}=-L_{31}=-C_{1} \frac{\partial}{\partial x_{1}}, \\
L_{23}=-L_{32}=-C_{2} \frac{\partial}{\partial x_{2}}, & L_{33}=C_{1} \frac{\partial^{2}}{\partial x_{1}^{2}}+C_{2} \frac{\partial^{2}}{\partial x_{2}^{2}} .
\end{array}
$$

\section{FUNDAMENTAL SOLUTION}

The fundamental solutions of the orthotropic thick plate taking into account the transverse shear deformation are a set of particular solutions of the differential Eq. (8) under a unit concentrated load, i.e., the solutions satisfy the following inhomogeneous differential equations:

$$
L_{i j}^{a d j} U_{k j}^{*}(\zeta, x)=-\delta(\zeta, x) \delta_{k i}
$$

in which $\delta(\zeta, x)$ denotes the Dirac delta function, $\zeta$ represents the source point, $x$ is a field point and $L_{i j}^{a d j}$ is the adjoint operator. Following Hörmander's operator method, the solutions of Eq. (7) can be written as:

$$
U_{k j}^{*}(\zeta, x)={ }^{c o} L_{j k}^{a d j} \phi(\zeta, x) .
$$

where $\phi(\zeta, x)$ is a unknown scalar function and ${ }^{c o} L_{j k}^{a d j}$ is the cofactor matrix of the operator $L_{j k}^{a d j}$ that is given by:

$$
\begin{aligned}
{ }^{c o} L_{\alpha \beta}^{a d j}= & E_{\alpha \beta} \nabla^{2} \nabla_{k}^{2}-B_{\alpha \beta} \frac{\partial^{2}}{\partial x_{\alpha} \partial x_{\beta}}-C_{1} C_{2} \frac{\partial^{2}}{\partial x_{\alpha} \partial x_{\beta}} . \\
{ }^{c o} L_{3 \alpha}^{a d j}= & -{ }^{c o} L_{\alpha 3}^{a d j}=\frac{\partial}{\partial x_{\alpha}}\left(E_{\alpha 3} \frac{\partial^{2}}{\partial x_{2}^{2}}+B_{\alpha 3} \frac{\partial^{2}}{\partial x_{1}^{2}}-C_{1} C_{2}\right) . \\
{ }^{c o} L_{33}^{a d j}= & D_{1} D_{k} \frac{\partial^{4}}{\partial x_{1}^{4}}+\left(D_{1} D_{2}-D_{1}^{2} \mu_{y x}^{2}-2 D_{1} D_{k} \mu_{y x}\right) \frac{\partial^{4}}{\partial x_{1}^{2} \partial x_{2}^{2}} . \\
& +D_{2} D_{k} \frac{\partial^{4}}{\partial x_{2}^{4}}-\left(D_{1} C_{2}+C_{1} D_{k}\right) \frac{\partial^{2}}{\partial x_{1}^{2}}-\left(C_{1} D_{2}+C_{2} D_{k}\right) \frac{\partial^{2}}{\partial x_{2}^{2}}+C_{1} C_{2} .
\end{aligned}
$$

The following symbols have been introduced:

$$
\begin{array}{ccc}
E_{11}=D_{2}, & E_{22}=D_{1}, & E_{12}=E_{21}=0, \\
B_{11}=D_{2}-D_{k}, & B_{22}=D_{1}-D_{k}, & B_{12}=B_{21}=\left(D_{1} \mu_{y x}+D_{k}\right), \\
E_{13}=C_{1} D_{2}-C_{2}\left(D_{1} \mu_{y x}+D_{k}\right), & B_{13}=C_{1} D_{k}, & E_{23}=C_{2} D_{k}, \\
B_{23}=C_{2} D_{1}-C_{1}\left(D_{1} \mu_{y x}+D_{k}\right), & \nabla_{k}^{2}=C_{1} \frac{\partial^{2}}{\partial x_{1}^{2}}+C_{2} \frac{\partial^{2}}{\partial x_{2}^{2}}, & \nabla^{2}=\frac{\partial^{2}}{\partial x_{1}^{2}}+\frac{\partial^{2}}{\partial x_{2}^{2}} .
\end{array}
$$


By substituting Eq. 11 into 7, we obtain the following equation:

$$
\begin{aligned}
& \left\{\nabla_{k}^{2}\left[D_{1} D_{k} \frac{\partial^{4}}{\partial x_{1}^{4}}+\left(D_{1} D_{2}-D_{1}^{2} \mu_{y x}^{2}-2 D_{1} D_{k} \mu_{y x}\right) \frac{\partial^{4}}{\partial x_{1}^{2} \partial x_{2}^{2}}+\right]\right. \\
& +D_{2} D_{k} \frac{\partial^{4}}{\partial x_{2}^{4}}-C_{1} C_{2}\left[D_{1} \frac{\partial^{2}}{\partial x_{1}^{2}}+2\left(2 D_{k}+D_{1} \mu_{y x}\right) \frac{\partial^{4}}{\partial x_{1}^{2} \partial x_{2}^{2}}\right. \\
& \left.\left.+D_{2} \frac{\partial^{4}}{\partial x_{2}^{4}}\right]\right\} \Phi(\zeta, x)=-\delta(\zeta, x)
\end{aligned}
$$

The derivation of the fundamental solution of Eq. (7) is reduced to that of Eq. (16). As soon as the solution of Eq. (16) is obtained, substituting it into Eq. (11) and by differentiation we can get the solutions of Eq. (7). Eq. (16) is a sixth order partial differential equation. Using the plane wave decomposition method, the partial differential Eq. (16) can be reduced to an ordinary differential equation, which simplifies the treatment of the problem. We first expand $\delta(\zeta, x)$ into a plane wave (see, for example, [8]):

$$
\delta(\zeta, x)=-\frac{1}{4 \pi^{2}} \int_{0}^{2 \pi}\left|\omega_{1}(x-\zeta)+\omega_{2}(y-\eta)\right|^{-2} d \theta
$$

in which $\left(\omega_{1}, \omega_{2}\right)$ are the coordinates of a point on the unit circle, i.e., $\omega_{1}=\cos (\theta), \omega_{2}=$ $\sin (\theta),(x, y)$ and $(\zeta, \eta)$ are the coordinates of a field point and a source point, respectively. Similarly, $\phi(\zeta, x)$ can be written as:

$$
\Phi(\zeta, x)=\int_{0}^{2 \pi} \varphi(\rho) d \theta
$$

where $\rho=\omega_{1}(x-\zeta)+\omega_{2}(y-\eta), \varphi(\rho)$ is a function depending only on $\rho$.

By substituting Eq. (17) and Eq. (18) into Eq. (16), and considering differential relationship $\frac{\partial}{\partial x_{\alpha}}=\omega_{\alpha} \frac{d}{d \rho}$, we obtain the following equation:

$$
\frac{d^{4}}{d \rho^{4}}\left(\frac{d^{2}}{d \rho^{2}}-p^{2}\right) \varphi(\rho)=\frac{1}{4 \pi^{2} a^{2}}|\rho|^{-2}
$$

in which

$$
\begin{aligned}
& a^{2}=C_{1} D_{1} D_{k} \omega_{1}^{6}+C_{1}\left(D_{1} D_{2}-D_{1}^{2} \mu_{y x}^{2}-2 D_{1} D_{k} \mu_{y x}\right) \omega_{1}^{4} \omega_{2}^{2}+C_{1} D_{2} D_{k} \omega_{1}^{2} \omega_{2}^{4}+C_{2} D_{1} D_{k} \omega_{1}^{4} \omega_{2}^{2}+ \\
& C_{2} D_{2} D_{k} \omega_{2}^{6} \\
& +C_{2}\left(D_{1} D_{2}-D_{1}^{2} \mu_{y x}^{2}-2 D_{1} D_{k} \mu_{y x}\right) \omega_{1}^{2} \omega_{2}^{4}, \\
& b^{2}=C_{1} C_{2}\left[D_{1} \omega_{1}^{4}+2\left(2 D_{k}+D_{1} \mu_{y x}\right) \omega_{1}^{2} \omega_{2}^{2}+D_{2} \omega_{2}^{4}\right] \text {, } \\
& p^{2}=b^{2} / a^{2} \text {. }
\end{aligned}
$$

The solution of Eq. (16) is now reduced to solve the ordinary differential Eq. (19). After four times integration of Eq. (19) and leaving out the constants of integration, we obtain:

$$
\frac{d^{2} \varphi(\rho)}{d \rho^{2}}-p^{2} \varphi(\rho)=-\frac{1}{8 \pi^{2} a^{2}} p^{2} \ln |\rho|
$$

The solution of Eq. (20) can be written as follows:

$$
\varphi(\rho)=f_{1}(\rho) \exp (p \rho)+f_{2}(\rho) \exp (-p \rho) .
$$


By the method of variation of parameters, the coefficients $f_{1}(\rho)$ and $f_{2}(\rho)$ can be obtained. By substituting $f_{1}(\rho)$ and $f_{2}(\rho)$ into Eq. (21), we obtain:

$$
\begin{array}{r}
\varphi(\rho)=\frac{1}{8 \pi^{2} p^{4} a^{2}}\left[p^{2} \rho^{2} \ln |\rho|+2 \ln |\rho|+3\right. \\
+\exp (p \rho) \int_{\rho}^{\infty} \frac{\exp (-p \sigma)}{\sigma} d \sigma \\
\left.-\exp (-p \rho) \int_{-\infty}^{\rho} \frac{\exp (p \sigma)}{\sigma} d \sigma\right] .
\end{array}
$$

Substituting Eq. (22) into Eq. (18) and integrating, we can obtain the function $\Phi(\zeta, x)$. The generalized displacement and boundary tractions can be expressed in the following forms:

$$
\begin{aligned}
U_{i j}^{*}(\zeta, x) & =\int_{0}^{2 \pi} \tilde{U}_{i j}^{*}(\rho) d \theta, \\
P_{i j}^{*}(\zeta, x) & =\int_{0}^{2 \pi} \tilde{P}_{i j}^{*}(\rho) d \theta .
\end{aligned}
$$

Details of the implementation of Eq. (23) and Eq. (24) can be found in [8].

\section{BOUNDARY INTEGRAL EQUATIONS}

The boundary integral equation of the orthotropic thick plates taking into account the transverse shear deformation is given by:

$$
c_{i j}(\zeta) U_{j}(\zeta)+f_{\Gamma} P_{i j}^{*}(\zeta, x) U_{j}(x) d \Gamma=\int_{\Gamma} U_{i j}^{*}(\zeta, x) P_{j}(x) d \Gamma+\int_{\Omega} q(x) U_{i 3}^{*}(\zeta, x) d \Omega
$$

where $\zeta, x \in \Gamma$ are source point and field point, respectively. The value of $c_{i j}(x)$ is equal to $\delta_{i j} / 2$ when $x$ is located on a smooth boundary. Equation (25) represents three integral equations, two $(i=\alpha=1,2)$ for rotations and one $(i=3)$ for deflection.

Bending moments at any internal point $\zeta$ can be computed by differentiating Eq. (25) with respect to the coordinate of the source point $\zeta$ and then substituting in Eq. (7) and Eq. (11) to give:

$$
M_{\alpha \beta}=\int_{\Gamma} U_{\alpha \beta k}^{*}(\zeta, x) p_{k} d \Gamma(x)-\int_{\Gamma} P_{\alpha \beta k}^{*}(\zeta, x) u_{k}(x) d \Gamma(x)+q \int_{\Gamma} W_{\alpha \beta}^{*}(\zeta, x) d \Gamma(x)
$$

The last integrals on the right hand side of equation (25) and (26), that are domain integrals, were transformed into boundary integrals by the radial integration method (see [12]). where the kernels $U_{i j k}^{*}, P_{i j k}^{*}$ and $W_{i j k}^{*}$ are computed following [13] but extending the formulation for the orthotropic case. 


\section{NUMERICAL RESULTS}

Consider a square clamped-plate under uniformly distributed load with amplitude $q=$ $-2.07 \times 10^{6} \mathrm{~N} / \mathrm{m}^{2}$. The plate is orthotropic with the following material properties: $E_{y}=$ $0.6895 \times 10^{10} \mathrm{~Pa}, E_{x}=2 \times E_{y}$ and $\nu=0.25$. The edges of plate is $a=0.254 \mathrm{~m}$ and thickness $h=0.0127 \mathrm{~m}$. This problem has been analysed by [11]. Constant boundary elements with equal length were used in the discretization.

As can be seen in Table 1, results are in good agreement with literature. Compared with results of [11], it was obtained a difference of $1.79 \%$ for the displacement at the central point and $0.002 \%$ for moment in $x$ direction at central point.

Table 1: Displacement and Moment at the center of plate.

\begin{tabular}{|c|c|c|}
\hline $\mathrm{NE}$ & Displacement $(\mathrm{m})$ & Moment $(\mathrm{N} \times \mathrm{m} / \mathrm{m})$ \\
\hline 40 & $6.94 \times 10^{-3}$ & $4,119.3$ \\
\hline 80 & $6.84 \times 10^{-3}$ & $4,088.5$ \\
\hline$[11]$ & $6.72 \times 10^{-3}$ & $4,089.5$ \\
\hline
\end{tabular}

\section{CONCLUSIONS}

This paper presented a boundary element formulation for the computation of moments at internal points. The moment integral equation was obtained by computing the derivative of the displacement integral equation with respect to the source point position. Derivatives of fundamental solutions were computed and the domain integral due to the distributed load is transformed into boundary integral by the radial integration method. So, all integrals in the formulation are boundary integrals. The obtained formulation were applied to a orthotropic square plate under uniformly distributed load. The moment and displacement at the central point of the plate were compared with literature showing good agreement. 


\section{REFERENCES}

[1] Weeën, F.V., Other B.B., "Application of the boundary integral equation method to Reissner's plate model". Journal. Num. Meth. Eng. v.18, n.1, 1-10, 1982.

[2] Long, S.Y., Brebbia, C.A., Telles, J.C.F., "Boundary element bending analysis of moderately thick plates". Comp. Mech. Publications v.5, n.8, 64-74, 1988.

[3] Karam, V.J., Telles, J.C.F., "On boundary elements for Reissner's plate theory”. Eng. Analysis v.5, n.1, 21-27, 1988.

[4] Ribeiro, G.O., "Sobe a formulao do mtodo dos elementos de contorno para a flexo de placas usando as hipteses de Reissner.". Escola de Engenharia de So Carlos, Universidade de So Paulo - USP, Tese (Doutorado) p.266, 1992.

[5] Hörmander, H., "Linear partial differential operators". Berlin: Springer Verlag, 1963.

[6] Abramowitz, M., Stegun, I.A., "Handbook of mathematical functions". New York: Dover Publications, 1965.

[7] De Barcellos, C.S., Silva, L.H.M., "A boundary element formulation for the Mindlin's plate model". Boundary Element Tecniques: Applications in Stress Analysis and Heat Transfer, Southampton, 1987.

[8] Wang J., Huang M., "Boundary element method for orthotropic thick plates".Acta Mechanica Sinica 7, 258-266, 1991.

[9] Sanches, L.C.F., "Uma resoluo de placas com a teoria de Mindlin atravs do mtodo dos elementos de contorno".Faculdade de Engenharia Civil, Universidade de Campinas, Dissertao (Mestrado) p.207, 1998.

[10] Useche, J.F., "Anlise pelo mtodo dos elementos de contorno de placas de Reissner trincadas e reparadas com compsitos colados".Faculdade de Engenharia Mecnica, Universidade de Campinas, Tese (Doutorado) p.165, 2007.

[11] Sladek J., Sladek V., Zhang Ch., Krivacek J. and Wen P.H., "Analysis of orthotropic thick plates by meshless local Petrov-Galerkin (MLPG) method". Int. J. Numer. Meth. Engn. 67, 1830-1850, 2006.

[12] Gao, X. "The radial integration method for evaluation of domain integrals with boundary only discretization". Engineering Analysis with Boundary Elements, 26, 905-916, 2002.

[13] Rashed Y.F., "Boundary method formulations for thick plates".Topics in Engineering, W.I.T. V.35, Publications Inc., 1999. 